\title{
Autosomal recessive lethal mutations in two mutator stocks of Drosophila ananassae
}

\author{
Muneo Matsuda \\ Department of Biology, School of Medicine, Kyorin University \\ 6-20-2 Shinkawa, Mitaka, Tokyo 181, Japan
}

(Received 7 September 1991)

\begin{abstract}
The frequency of recessive lethals in the 2 nd chromosome was examined in two mutator stocks of Drosophila ananassae, $c a$ and $c a ; p x$. They are characterized respectively by possessing an extrachromosomal clastogenic mutator in males, and by the retrotransposon "tom", which induces $\mathrm{Om}$ mutability only in females. The frequencies of recessive lethal mutations in the 2nd chromosome among progenies from males and females of the $c a ; p x$ stock are 0.35 and 0.34 percent, respectively. Similarity of these frequencies indicates that tom does not induce recessive lethals in females. In contrast to the $c a ; p x$ stock, the frequency of recessive lethals in males of the $c a$ mutator stock was estimated to be 1.54 percent for the 2nd chromosome. No visible mutants except Minutes were recovered. Some recessive lethals derived from $c a$ stock males were associated with chromosomal rearrangements. Being consistent with its high rate of $\mathrm{Mi}$ nute mutation it was demonstrated that the $c a$ clastogenic mutator also induced recessive lethals.
\end{abstract}

\section{INTRODUCTION}

Based on his frequent recovery of spontaneous visible mutants, Kikkawa (1938) suggested that Drosophila ananassae may be characterized by high mutability. Kikkawa (1938) also found a translocation in a Ryukyu wild stock and this has been followed by frequent reports of rearrangements such as reciprocal translocations, pericentric inversions or deficiencies that are not ordinarily reported to occur or persist in natural populations of other Drosophila (Dobzhansky and Dreyfus, 1943; Freire-Maia, 1961; Futch, 1966; Ray-Chaudhuri and Jha, 1966; Nirmala and Krishnamurthy, 1970, 1972; Sreerama and Krishnamurthy, 1970; Singh et al., 1971; Tomimura, personal communication). In laboratory stocks, Hinton $(1979,1981,1983,1984)$ identified four highpermutability systems. In the bri and pc marker stocks, two distinct, dominant mutators linked to the 3rd chromosome were diagnosed along with specific chromosomal (bri) or extrachromosomal $(p c)$ suppressors. These cryptic mutability of the stocks were uncovered only in outcrosses. In the $c a$ marker stock, an extrachromosomally transmitted clastogenic mutator responsible for chromosome damage in sperm was found to be accompanied by a genetic (or cytotypic) suppressor system. A $c a ; p x$ 
derivative of the $c a$ stock, synthesized by outcrossing with a $p x$ stock, lacks the clastogenic mutator, but is characterized by hypermutability in which dominant eye morphology $(\mathrm{Om})$ mutants are recovered almost exclusively. These mutants have been attributed to insertion of a retrotransposon named tom (Shrimpton et al., 1986; Tanda et al., 1988 and 1989).

The $c a$ mutator system was defined primarily by assaying the frequencies of dominant visible, recessive lethal Minutes bristle mutations, which were often associated with deficiencies. Direct cytological screens for chromosome rearrangements among larvae as well as the incidence of dominant lethality among embryos confirmed the clastogenic effect of the $c a$ mutator on paternal chromosomes introduced into suppressor-free egg cytoplasm (Hinton, 1981). The mutations of the $c a$ mutator system appear among the progeny of outcrossed $c a$ males. $O m$ mutations originate exclusively in the gametes of $c a ; p x$ females, being invariably free of rearrangements. The frequency of Minutes was not significantly elevated in this stock (Hinton, 1984). There is, none the less, at least one feature common to the two systems in that the extrachromosomal mutator induced breakages and the retrotransposon induced $\mathrm{Om}$ mutations are restricted to euchromatic chromosome segments.

In view of the foregoing comparisons of these mutator stocks that share their genetic background, it is of interest to compare them with respect to another measure of mutability, namely recessive lethals.

\section{MATERIALS AND METHODS}

The simplest and most common method of detecting recessive lethal mutations in $D$. melanogaster is to recover them as heterozygotes over an $\mathrm{X}$ chromosome balancer. In $D$. ananassae, there is no suitable balancer for the $\mathrm{X}$ chromosome, so recessive lethals must be detected on the 2nd chromosome. From a natural population in Papua New Guinea, Futch (1968) isolated and described a multiple inverted 2nd chromosome, designated Ins $(2 L+2 R)$ NG2 and abbreviated NG2. This chromosome effectively eliminates recombination throughout the 2nd chromosome in standard(+)/NG2 flies. NG2 carries at least one recessive lethal itself. Two NG2 balancer stocks were used: NG2 $e^{D} / r s$ and NG2/ca M(2)665. $e^{D}$ is ebony dominant (body color) and $r s$ is a recessive rose eye color mutant with cause recessive female-sterile located near the tip of $2 \mathrm{R} . \quad c a$ is claret eye color and $M(2) 665$ is the Minutes bristle mutant in 2L. Both the $c a$; $p x$ and $c a$ mutator stocks carried standard sequences in the X, 2nd and 3rd chromosomes.

Outcrosses of males from the $c a$ stock, or of males and females from the $c a ; p x$ stock, to flies from the $r s / N G 2 e^{D}$ stock produced $F_{1}$ flies which were scored systematically for Minutes and casually for any other dominant visible mutants. Among the $\mathrm{F}_{1}$ progeny, non-Minutes $\mathrm{F}_{1}$ females of the $c a^{*} / \mathrm{NG} 2 e^{D}$ genotype were collected and mated singly to $c a M(2) 665 / \mathrm{NG} 2$ males to produce $c a^{*} / \mathrm{NG} 2$ 
flies sharing identical $c a^{*}$ chromosomes. Upon inbreeding $c a^{*} / \mathrm{NG} 2$ daughters and sons of the same $\mathrm{F}_{1}$ females, the presence or absence of claret $\left(c a^{*} / c a^{*}\right)$ progeny revealed the absence or presence of a recessive lethal in that $c a^{*}$ chromosome. Each $c a^{*}$ chromosome identified with lethal was maintained in a $c a^{*} l / \mathrm{NG} 2$ stock until completion of complementation tests identifying allelic lethals. In addition, polytene chromosomes of larvae from some lethal stocks were examined for any associated rearrangements.

Stocks and experimental matings were cultured in $25 \times 95 \mathrm{~mm}$ shell vials containing $10 \mathrm{ml}$ of medium composed of corn meal, brewers' yeast, molasses, agar and mold inhibitor. Experimental matings routinely used virgin adults aged three to four days and maintained at $25^{\circ} \mathrm{C}$.

\section{RESULTS}

Only two Minutes appeared among the 6796 progeny of the $c a ; p x$ stock males (Table 1). Among $868 c a^{*}$ chromosomes isolated via $\mathrm{F}_{1}$ females with $c a ; p x$ father and tested for homozygous viability, six bore recessive lethals that fell into 5 complementation groups (Table 2). Progeny from the $c a ; p x$ stock females, like their male cohorts, produced only two Minutes, and no Om mutants among 4210 $\mathrm{F}_{1}$ progeny scored. This result is not unexpected given the reported $\mathrm{Om}$ fre-

Table 1. Frequencies of Minutes in $\mathrm{F}_{1}$ from outcrosses of mutator stocks in D. ananassae

\begin{tabular}{|c|c|c|c|}
\hline Female Marents & $\begin{array}{l}\text { No. of progeny } \\
\text { counted }\end{array}$ & $\begin{array}{c}\text { No. of Minutes } \\
\text { observed }\end{array}$ & $\begin{array}{l}\text { Minutes frequency } \\
\text { per chromosome }\end{array}$ \\
\hline$N G \mathscr{2} e^{D} \times c a$ & 7374 & 26 & 0.00353 \\
\hline$N G \mathscr{2} e^{D} \times c a ; p x$ & 6769 & 2 & 0.00030 \\
\hline$c a ; p x \times N G 2 e^{D}$ & 4210 & 2 & 0.00048 \\
\hline
\end{tabular}

Table 2. Frequencies of 2-linked recessive lethals per chromosome in F1 from outcrosses of mutator stocks in $D$. anassae

\begin{tabular}{lcccc}
\hline \hline $\begin{array}{c}\text { Parents } \\
\text { Female Male }\end{array}$ & $\begin{array}{c}\text { No. of } \\
\text { chromosomes } \\
\text { isolated }\end{array}$ & $\begin{array}{c}\text { No. of } \\
\text { chromosomes } \\
\text { with } \\
\text { recessive } \\
\text { lethals }\end{array}$ & $\begin{array}{c}\text { No. of } \\
\text { complementation } \\
\text { groups of } \\
\text { recessive } \\
\text { lethals }\end{array}$ & $\begin{array}{c}\text { 2-linked } \\
\text { recessive } \\
\text { lethals } \\
\text { frequency }\end{array}$ \\
\hline NG2 $e^{D} \times c a$ & 988 & 29 & 15 & 0.0154 \\
NG2 $e^{D} \times c a ; p x$ & 868 & 6 & 5 & $(15 / 974)$ \\
$c a ; p x \times \mathrm{NG2} e^{D}$ & 590 & 10 & 4 & 0.0035 \\
$\left(3^{*} / 865\right)$ \\
0.0034 \\
$\left(2^{*} / 582\right)$
\end{tabular}

* see text 
quency of $2 / 10^{4}$ (Hinton 1984). A total of $590 \mathrm{ca}$ ( chromosomes analyzed yielded ten recessive lethals, that defined four complementation groups. Two of the complementation groups derived from $c a ; p x$ stock males were allelic to those forward the recessive lethals recovered from $c a ; p x$ stock females. The mutations in these two complementation groups are considered to be pre-existing lethals in the $c a ; p x$ stock, and these were omitted them for the calculations of the frequencies. The estimated frequencies of recessive lethal in the 2nd chromosome are $0.35(3 / 865)$ and $0.34(2 / 582)$ percent for chromosomes derived from outcrosses of $c a ; p x$ stock males and females, respectively. None of the lethals recovered from outcrosses of $c a$; $p x$ males or females were examined cytologically for chromosome rearrangements. However, all the five lethals showed independent assortment of the 2nd and 3rd chromosomes in testcrosses of $c a^{*} 1 / \mathrm{NG} 2 ;+1$ $p x$ males to $c a ; p x$ females.

From outcrosses of $c a$ stock males to $r s / N G 2 e^{D}$ females, examination of 7374 progeny uncovered 26 putative Minutes mutations (Table 1). This frequency (0.0035) is lower than the estimated frequency $(0.0104)$ by Hinton $(1981,1984)$ but statistically nonsignificant. From $\mathrm{F}_{1} c a^{*} / \mathrm{NG} 2 e^{D}$ females, $988 c a^{*}$ chromosomes were assayed and 29 carried recessive lethals. Complementation tests among these 29 lethals revealed 15 complementation groups (Table 2). Three lethal loci were represented by clusters of two, four, and eleven mutations each. When such clusters may reflect amplification of pre-meiotic lethals occurring in the parental males' germ line, the frequency of recessive lethals is 1.54 percent per chromosome (15/974). Among the 15 lethal loci, five were associated with chromosomal aberrations including two different pericentric and one paracentric inversions, one deficiency and one $\mathrm{X} ; 2$ translocation. All breakpoints were in euchromatin. Thus the frequency of chromosomal aberrations identified by recessive lethals was $0.51(5 / 974)$ percent.

\section{DISCUSSION}

ca ; px stock

The $c a ; p x$ stock was derived from the cross between $p x$ females and $c a$ males (Hinton, 1984). Therefore extrachromosomal mutator elements of the $c a$ stock were not transmitted to the new synthesized stock. The $p x$ stock is silent for Minutes mutation (Hinton, 1981). Both sexes of the $c a ; p x$ stock still have the property of Minutes mutability equivalent to that of the $p x$ original stock, whose Minutes frequency is 0.0006 (Hinton, 1981). This value is comparable to the data of this experiment. The $c a$; $p x$ stock is characterized by the $O m$ hypermutability system in which occur at high rate spontaneously eye morphology mutations, $\mathrm{Om}$ (Hinton, 1984). Om mutants are semi-dominant and arise exclusively in oocytes. They map to at least 25 loci (Hinton, 1984, 1988) in the $X$, 2nd and 3rd chromosomes. They are associated with the insertion of the tom retrotransposon 
(Shrimpton et al., 1986, Matsubayashi et al., 1986). No Om mutation was detected in the $\mathrm{F}_{1}$ generation bore in the present experiment, because of the small progeny number scored. In an unrelated experiment, $3 \mathrm{Om}$ mutations were recovered among 3333 progeny of $c a ; p x$ stock. Therefore I considered the $c a$; $p x$ stock still has an $O m$ hypermutability system. Minutes and other dominant visible mutations could seldom be found in $c a$; $p x$ stock (Hinton, 1984; present data). The present data shows that the frequency of the 2nd chromosomes carrying recessive lethals is not significantly different between females $(0.35 \%)$ and males $(0.34 \%)$ of the $c a ; p x$ stock. This is in exquisite contrast to $\mathrm{Om}$ mutability restricted to females (Hinton, 1984). The range of the lethal frequency in the 2nd chromosome, $0.34 \%$ to $0.35 \%$, is comparable to the recessive lethal mutation rates $(0.57 \%$ in males and $0.72 \%$ in females) in the 3rd chromosome of $D$. melanogaster (Wallace, 1968). The 2nd chromosome of D. ananassae corresponds to the 3rd chromosome of D. melanogaster (Paterson and Stone, 1954). $\mathrm{Om}$ mutation rate/generation/locus can be estimated to be $8.0 \times 10^{-6}$ by dividing the occurrence rate, $2 \times 10^{-4}$, by the number of $\mathrm{Om}$ loci, 25 . If tom insertion induces recessive lethal mutations as well as $\mathrm{Om}$ mutations throughout the genome in the $c a ; p x$ females, an excess of $1.6 \%\left(8.0 \times 10^{-6} \times 2000\right.$ loci) or 8 to 9 mutations over what are recovered in the $c a ; p x$ males would be expected for the 2nd chromosome in which 2000 loci are assumed. The number of loci on the 2nd chromosome was assumed by the hypothesis of one gene and one polytene band (Judd et al., 1972). In the present experiment only $0.34 \%, 2$ lethals among 582 chromosomes were found. Despite the fact that tom elements inserts into the target sequence TATAT (Tanda et al., 1988) which should be present in many sites in the genome and many tom elements found at sites other than $\mathrm{Om}$ loci (Shrimpton et al., 1986; Tanda et al., 1988), the present results indicate that the Om-tom mutability may not have a significant role in the production of recessive lethal mutations or dominant visible mutations except $O m$ mutations. Southern blot analyses of spontaneous complete revertants of $O m(1 D) 9$ and $O m(2 D) 63$ revealed that all tom insertions were lost without DNA rearrangements (Tanda et al., 1989; Matsubayashi et al., 1991). These findings and the present results indicate that tom transposition may not induce chromosome breakages, in contrast to the $c a$ mutator.

ca stock

The $c a$ stock has an extrachromosomal clastogenic mutator which produced high frequencies of chromosomal rearrangements associated with dominant lethals or visible Minute mutations among the progeny from outcrossed ca males or the $\mathrm{F}_{1}$ sons of outcrossed $c a$ females (Hinton 1979, 1981). The high frequency (1.54\%) of recessive lethals in the 2 nd chromosome was found in the progeny from $c a$ mutator stock males in this study. Clusters at three lethal loci were found among 15 lethal complementation groups. Although these clusters may be amplification 
of pre-meiotic lethals, it is possible that such clusters may reflect pre-existing lethals segregating in the $c a$ stock. These two possibilities can hardly be distinguished. However, high frequency of recessive lethals found in the present experiment indicates that $c a$ mutator males have many recessive lethals and/or newly arisen lesions in the sperms which could not be repaired in the eggs of balancer females. This result is consistent with the previous reports of Minute mutability induced by the $c a$ extrachromosomal mutator (Hinton, 1979, 1981).

Hinton $(1979,1981)$ showed that $c a$ extrachromosomal mutator produced $2.6 \%$ chromosome breakages among 639 progeny. In the present data, high frequency (33.3\%) of rearrangements was found among chromosomes carrying recessive lethals derived from $c a$ males, supporting the previous reports (Hinton, 1979, 1081). In Drosophila melanogaster, a high occurrence rate of chromosomal rearrangement has been found among progeny of the so-called dysgenic $\mathrm{F}_{1}$. Engels and Preston (1984) reported that the majority of breaks induced by $\mathrm{P}$ elements were intrachromosomal two-break events. Another transposable element, hobo, has been found to mediate the rearrangements restricted to a single chromosome arm in Uc (Unstable X chromosome) stock of D. melanogaster (Lim, 1988; Blackman and Gelbart, 1989), although it is still unclear whether hobo produces only intrachromosomal aberrations. In contrast to these systems in $D$. melanogaster, chromosomal rearrangements induced by ca-mutator are characterized as follows, 1) breakage events were generated during spermatogenesis of the $c a$ inbred stock, 2) both inter- and intra chromosome rearrangements were observed, 3) their breakpoints were located in euchromatin region, and 4) chromosomal lesions carried in sperm of $c a$ males were efficiently repaired by suppressor in fertilized eggs derived from $c a$ stock females (Hinton, 1981).

This work was suggested by, and initiated in the laboratory of, Claude W. Hinton, Department of Biology, The College of Wooster, Ohio, USA. The author expresses Drs. C. W. Hinton, C. H. Langley for their kind support throughout this work. I am grateful to Drs. C. H. Langley, M. M. Green and Y. N. Tobari for reading the manuscript and making numerous useful suggestions.

\section{REFERENCES}

Blackman, R. K. and Gelbart, W. M. (1989). The transposable element hobo of Drosophila melanogaster. In: Mobile DNA (eds.: D. E. Berg and M. M. Howe), pp. 523-529. American Society for Microbiology, Washington, DC.

Dobzhansky, Th. and Dreyfus, A. (1943). Chromosomal aberrations in Brazilian Drosophila ananassae. Proc. Natl. Acad. Sci. USA 29, 301-305.

Engels, W. R. and Preston, C. R. (1984). Formation of chromosome rearrangements by P factors in Drosophila. Genetics 107, 657-678.

Freire-Maia, N. (1961). Peculiar gene arrangements in Brazilian natural populations of Drosophila ananassae. Evolution 15, 486-495.

Futch, D. G. (1966). A study of speciation in South Pacific populations of Drosophila ananassae. Univ. Texas Publ. 6615, 79-120. 
Kikkawa, H. (1938). Studies on the genetics and cytology of Drosophila ananassae. Genetica 20, $458-516$.

Hinton, C. W. (1979). Two mutators and their suppressors in Drosophila ananassae. Genetics 92, 1153-1171.

Hinton, C. W. (1981). Nucleocytoplasmic relations in a mutator-suppressor system of Drosophila ananassae. Genetics 98, 77-90.

Hinton, C. W. (1983). Relation between factors controlling crossing over and mutability in males of Drosophila ananassae. Genetics 104, 95-112.

Hinton, C. W. (1984). Morphogenetically specific mutability in Drosophila ananassae. Genetics 106, 631-653.

Hinton, C. W. (1988). Formal relations between $O m$ mutants and their suppressors in Drosophila ananassae. Genetics 106, 631-653.

Hinton, C. W. and Downs, J. E. (1975). The mitotic, polytene, and meiotic chromosomes of Drosophila ananassae. J. Hered. 66, 353-361.

Judd, B. H., Shen, M. W. and Kaufman, T. C. (1972). The anatomy and function of a segment of the $\mathrm{X}$ chromosome of Drosophila melanogaster. Genetics 71, 139-156.

Lim, J. K. (1989). Intrachromosomal rearrangements mediated by hobo transposons in Drosophila melanogaster. Proc. Natl. Acad. Sci. USA 85, 9153-9157.

Matsubayashi, H., Juni, N., Usui, K., Hori, S. H. and Tobari, Y. N. (1991). Molecular and histological characterization of the $\mathrm{Om}(2 D)$ mutants in Drosophila ananassae. Mol. Gen. Genet. 227, 165-172.

Matsubayashi, H., Matsuda, M. and Tobari, Y. N. (1986). The transposable element tom, insertion mutation $\mathrm{Om}$, and suppressors of $\mathrm{O} m$ in Drosophila ananassae. Jpn. J. Genet. 61, 594. (abstr. in Japanese)

Moriwaki, D. and Tobari, Y. N. (1975). Drosophila ananassae. In: Handbook of Genetics, Vol. 3 (ed.: R. C. King), pp. 513-535. Plenum, New York.

Nirmala, S. S. and Krishnamurthy, N. B. (1970). Report on two new translocations in a natural population of Drosophila ananassae from Hiriyur (Mysore State, India). Dros. Inf. Serv. 45,166-318.

Nirmala, S. S. and Krishnamurthy, N. B. (1972). New gene arrangements in Drosophila ananassae. Dros. Inf. Serv. 48, 103-104.

Patterson, J. T. and Stone, W. S. (1954). Evolution in the Genus Drosophila. Macmillan, New York.

Ray-Chaudhuri, S. P. and Jha, A. P. (1966). Genetics of natural populations of Drosophila ananassae (Abstr.). Proc. Ind. Sci. Cong. Assoc. 53, 317-318.

Shrimpton, A. E., Montgomery, E. A. and Langley, C. H. (1986). Om mutations in Drosophila ananassae are linked to insertions of a transposable element. Genetics 114, 125-135.

Singh, V. K., Mishra, M. and Jha, A. P. (1971). A new pericentric inversion in Drosophila ananassae. Dros. Inf. Serv. 48, 97.

Sreerama, R. G. and Krishnamurthy, N. B. (1972). Two new gene arrangements in Drosophila ananassae from South India. Dros. Inf. Serv. 48, 140-142.

Tanda, S., Shrimpton, A. E., Ling-Ling, C., Itayama, H., Matsubayashi, H., Saigo., K., Tobari, Y. N. and Langley, C. H. (1988). Retrovirus-like features and site specific insertions of a transposable element, tom, in Drosophila ananassae. Mol. Gen. Genet. 214, 405-411.

Tanda, S., Shrimpton, A. E., Hinton, C. W. and Langley, C. H. (1989). Analysis of the $O m(1 D)$ locus in Drosophila ananassae. Genetics 123, 495-502.

Wallace, B. (1968). Mutation rates for autosomal lethals in Drosophila melanogaster. Genetics 60, 389-393. 